\title{
Development and Characterization of Cephradine Proniosomes for Oral Controlled Drug Delivery
}

\author{
Muhammad Adnan Faisal Saim ${ }^{1}$, Lubna Bashir ${ }^{1}$, Shazia Naz', Sana Ghayas ${ }^{2, *}$, Rabia Bushra ${ }^{2}$, \\ Zubair Anwar ${ }^{3, \star}$, Syed Akif Uddin ${ }^{1}$, Ubedullah Korai ${ }^{1}$
}

1'Department of Pharmaceutics, Faculty of Pharmacy, Federal Urdu University of Arts, Science and Technology, Gulshan Campus, Karachi, PAKISTAN.

${ }^{2}$ Faculty of Pharmaceutical Sciences, Dow College of Pharmacy, Dow University of Health Science, Karachi, PAKISTAN.

${ }^{3}$ Baqai Institute of Pharmaceutical Sciences, Baqai Medical University, Super Highway, Gadap Road, Karachi, PAKISTAN.

\begin{abstract}
The role of Proniosomes in oral controlled drug delivery system is well accepted. Presently, an attempt was made to develop cephradine (CP) Proniosomes to prolong its duration of action with better efficacy. Overall, eighteen formulation trials were developed using variable quantities of sorbitol (FOS-F8S) and maltodextrin (FOM-F8M) carriers along with span60 and cholesterol. Trials were evaluated for powder flowability, drug entrapment efficiency and in vitro drug release. Based on the mentioned characteristics, formulations F4M and F4S were optimized. Stability test was performed on optimized Proniosomes for three months at temperature $\left(2-8^{\circ} \mathrm{C}\right)$. Comparison for antimicrobial sensitivity against Staphylococcus aureus was also made between optimized and marketed conventional $\mathrm{CP}$ capsule. More than $80 \%$ drug release was observed in $22 \mathrm{hr}$ in the trial formulations. The optimized CP Proniosomes were found to be highly stable with \% drug entrapment efficiency of $78.60 \pm 0.15$ and $88.41 \pm 0.19$ respectively for F4M and F4S. The prepared Proniosomes possessed higher bactericidal activity against $S$. aureus than reference products (M1 and M2). In conclusion, CP Proniosomes have been successfully prepared using sorbitol/maltodextrin carrier. Sorbitol carrier exhibited marginally better drug entrapment efficiency and bactericidal activity than the maltodextrin trial and marketed brands. This effort offers improved drug delivery with the potential of more effective controlled therapy.
\end{abstract}

Key words: Proniosomes, Cephradine, Nano-carriers, Niosomes, Bactericidal activity, Controlled drug delivery.

\section{INTRODUCTION}

Conventional pharmaceutical dosage forms are unable to control the drug delivery for longer duration in the body. ${ }^{1}$ They are also associated with the problem of dosing interval resulting in large peaks and valleys in the drug plasma profiles. ${ }^{2}$ Moreover, patients' compliance with multiple dosing regimens and drug related adverse effects (especially for potent drugs) are major concerns in clinical setting. ${ }^{3}$ However, these problems may overcome by designing the controlled and targeted delivery systems ${ }^{4}$ owing to improve overall treatment efficiency along with lower incidence of adverse effect. Unfortunately, very limited drug delivery system behaves ideally to meet the pharmaceutical and clinical goals. In this regard, many attempts are being made to achieve such targets through novel drug delivery approaches. ${ }^{5,6}$ Vesicular drug delivery is consider to be one among them, improving the bioavailability of medicinal agents, especially poorly soluble moieties. Hydrophilic and lipophilic drugs could be developed into sustained release vesicular systems to overcome the rapid drug elimination of highly metabolizable medicinal components. Consequently, addressing the problems related to drug solubility, stability and degradation. ${ }^{6}$
Submission Date: 02-03-2021; Revision Date: 09-09-2021; Accepted Date: 04-10-2021.

DOI: 10.5530/ijper.56.1s.44 Correspondence: Dr. Zubair Anwar, Pharm. D. M. Phil. Ph. D. NCN Fellow, Poland, Assistant Professor, Department of Pharmaceutical Chemistry, Baqai Institute of

Pharmaceutical Sciences, Baqai Medical University, Karachi, PAKISTAN.

E-mail: zubair_ana@hotmail. com

Dr. Sana Ghayas Dow College of Pharmacy Dow Medical University, Karachi, PAKISTAN.

Email: sana.ghayas@duhs. edu.pk

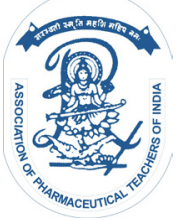

www.ijper.org 
Several vesicular drug delivery systems including liposomes, niosomes, transferosomes, pharmacosomes and provesicular systems (proliposomes and Proniosomes) have been investigated. ${ }^{7}$ Niosomes and Proniosomes are advantageous due to improved solubility, skin adhesion and penetration with drug release sustainability. Proniosomes also showed great versatility in lipophilic and hydrophilic drug moieties encapsulation. Unfortunately, physical instability is a critical concern in designing and implementation. ${ }^{8}$ This type of systems not only required an appropriate carrier to protect the drug from rapid degradation and clearance. Rather, selection and preference of non-ionic surfactants over the cationic, anionic and ampholytic surfactants is also quite challenging in formulation development of Proniosomes.,10 Various drug deliveries by different route of administration are possible using Proniosomes based niosomes such as ophthalmic, topical, parenteral and oral etc. ${ }^{11,12}$

Presently, no encapsulated cephradine (CP) Proniosomes is available in the local market. The study aimed to fabricate vesicular system of Cepheradine using various combinations of non-ionic surfactant (span 60), membrane stabilizer (cholesterol) and coating carriers (sorbitol, maltodextrin). Influence of these additives on the quality attributes including flowability, drug entrapment efficiency, drug release profile and stability will also be accessed. Moreover, microbiological comparison will be made between optimized CP Proniosomes and the conventional marketed capsules. Henceforth, this study is supportive in developing stable and effective sustained release dosage forms for different therapeutic agents/drugs.

\section{MATERIALS AND METHODS Materials}

CP pure with purity $99.7 \%$ on dried basis, batch number F1BCO2172W and expiry date 18/ 12/ 2021 was gifted by Opal Laboratory Karachi, Pakistan. Cholesterol, sorbitol and lactose were provided by Venus Pharma, Lahore, Otsuka Hub, Balochistan and Aventis Pharma Karachi, Pakistan, respectively. Staphylococcus aureus ATCC $\mathbb{}$ 25923, tryptone soya broth (CM129-OXOID) and span 60 (sorbitan monostearate) were obtained from Sigma Aldrich (St. Louis, MO, USA). Maltodextrin was procured from Grain Processing Corporation (NY, USA). Phosphate buffer saline, hydrochloric acid, potassium chloride, chloroform, ethanol, methanol were procured from Sigma- Aldrich (analytical grade).

\section{Instruments}

Rotary evaporator (Lab-scale, REV-1001), water bath (Memmert, WTB), sonicator (Thmos Scientific, USA), desiccator (Thmos Scientific, USA), centrifuge (Thmos Scientific, USA), optical microscope (LCD Digital Microscope, Thmos Scientific, USA), dissolution apparatus (Erweka DT 700, Husenstamm, Germany), spectrophotometer (Shimadzu UV-1601), electronic balance (AUW220, UNI Blog, Shimadzu Corp.)

\section{Methods}

\section{Preparation of CP Proniosomes}

Proniosomes were developed with some modifications in the previously reported method. ${ }^{13,14}$ The stock solution was prepared using defined quantities (Table 1) of cholesterol, and CP in $10 \mathrm{ml}$ of chloroform and ethanol $(1: 1, \mathrm{v} / \mathrm{v})$ solvent system. A suitable quantity of prepared stock solution was transferred to $100 \mathrm{ml}$ round bottom flask containing carrier (maltodextrin/sorbitol) (Table 1). Further, span-60 was added to the mixture during the processing to lower the surfactant loading. Excess of solvent was evaporated using a rotary evaporator $(60 \mathrm{rpm})$, temperature of $45 \pm 2^{\circ} \mathrm{C}$ and at a pressure of $600 \mathrm{mmHg}$ till the dry powder is obtained. This dried powder referred to be "proniosomes", was then placed in a desiccator overnight to ensure complete moisture removal from powder proniosomes. These powdered samples were stored in the airtight containers (glass vials) at temperature of $2-8^{\circ} \mathrm{C}$.

\section{Study variables}

$\mathrm{CP}$ proniosomes were prepared with variable quantities of carriers including sorbitol (F0S to F8S) and maltodextrin (F0M to F8M) along with cholesterol and span-60 blend. Effect of formulation variables including surfactant (span-60), carriers (maltodextrin/sorbitol) and membrane stabilizer (cholesterol) were observed on

\begin{tabular}{|c|c|c|c|c|c|c|}
\hline $\begin{array}{l}\frac{0}{z} \\
\text { i }\end{array}$ & 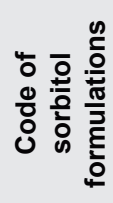 & 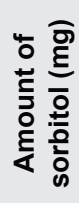 & 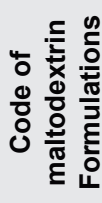 & 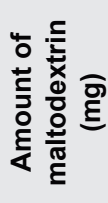 & 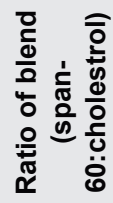 & 을 \\
\hline 1 & FOS & 300 & FOM & 300 & $300: 0$ & \multirow{9}{*}{300} \\
\hline 2 & F1S & 270 & $\mathrm{~F} 1 \mathrm{M}$ & 270 & $270: 30$ & \\
\hline 3 & F2S & 240 & $\mathrm{~F} 2 \mathrm{M}$ & 240 & $240: 60$ & \\
\hline 4 & F3S & 210 & F3M & 210 & $210: 90$ & \\
\hline 5 & F4S & 180 & F4M & 180 & $180: 120$ & \\
\hline 6 & F5S & 150 & F5M & 150 & $150: 150$ & \\
\hline 7 & F6S & 120 & F6M & 120 & $120: 180$ & \\
\hline 8 & F7S & 90 & F7M & 90 & $90: 210$ & \\
\hline 9 & F8S & 60 & F8M & 60 & $60: 240$ & \\
\hline
\end{tabular}


the response variables such as morphological appearance, flowability, drug entrapment efficiency and drug release profile. Formulation optimization of proniosomal trials was performed on the basis of above characterization parameters.

\section{Hydration and preparation of niosomes}

The proniosomes were hydrated at $60-65^{\circ} \mathrm{C}$ in a water bath for $10 \mathrm{~min}$. Aqueous phase was later added to adjust the volume. The resultant niosomal gel was sonicated for 30 sec at room temperature. ${ }^{14}$

\section{Microscopic evaluation}

Proniosomes and niosomal dispersion were examined by optical microscope to observe the vesicular structures both in dried and hydrated forms. Magnification of 40X was used and images were captured using attached camera device.

\section{Drug entrapment efficiency}

The entrapment efficiency of proniosomes was estimated by indirect centrifugation method..$^{15}$ One milliliter of drug dispersion (niosomes) was centrifuged at $4000 \mathrm{rpm}$ for 40min. The supernatant was taken out, diluted with phosphate buffer and analyzed using UV spectrophotometer at $254 \mathrm{~nm}$ wavelength. ${ }^{16}$ The entrapment efficiency of the vesicles was determined by subtracting the amount of unentrapped drug from the total drug ${ }^{17}$ using following formula:

Entrapment Efficiency $(\mathrm{EE} \%)=\frac{\text { Amount of Drug Entrapped }}{\text { Total Amount of Drug }} \times 100$

\section{Flowability}

The flowability of cepharadine proniosomes was accessed by angle of repose using fix funnel method. The angle of repose was calculated using below equation:

$$
\tan (\theta)=\text { height } / 0.5 \text { base }
$$

\section{Dissolution study}

The release of $\mathrm{CP}$ from the proniosome powders (F0S-F8S, F0M-F8M) was evaluated as documented previously. ${ }^{13}$ The drug release was conducted using USP dissolution apparatus-I (rotating basket assembly) at $37 \pm 0.5^{\circ} \mathrm{C}$ and at a motion pace of $50 \mathrm{rpm}$. The drug release was carried out in $500 \mathrm{~mL}$ buffer $\mathrm{HCl}(\mathrm{pH} 1.2)$ for two hours and then switched to phosphate buffer saline (PBS) of $\mathrm{pH} 7.4$ for $24 \mathrm{hr}$. Samples of $2 \mathrm{~mL}$ was taken at different time intervals $(0-24 \mathrm{~h})$ with replenishment of equal volume with fresh medium. After filtration the samples in triplicate were analyzed spectrophotometrically at $254 \mathrm{~nm}$ wavelength. ${ }^{16}$

\section{Stability Testing}

$\mathrm{CP}$ proniosomes exhibiting the best attributes ( $\mathrm{F} 4 \mathrm{~S}$ and F4M) were kept in glass vials at temperature of $5 \pm 3^{\circ} \mathrm{C}$ in refrigerator for three months. ${ }^{18}$ Samples were taken at time interval of $0,15,30,45,60$ and 90 days and assessed spectrophotometrically at $254 \mathrm{~nm}$ wavelength in triplate for $\%$ drug entrapment efficiency and $\%$ drug remaining. ${ }^{16}$

\section{Filling of optimized CP proniosomes in hard gelatin capsules}

Optimized proniosomes F4S and F4M were filled manually in HGC (12 mm), each containing equivalent amount of CP $250 \mathrm{mg}$. 19.2g of sorbitol proniosomes (F4S) with $78.89 \%$ entrapment were taken with $0.1 \%$ lactose (diluent) for powder mixture filling. Likewise, 16.8g of maltodextrin proniosomes (F4M) with $89.31 \%$ entrapment were used with $0.1 \%$ lactose for powder fill mixture.

\section{Pharmaceutical quality testing of proniosomes filled capsules}

Weight variation of proniosomes HGCs containing sorbitol (F4S) and maltodextrin (F4M) was performed as per BP recommendation. 10 units of each formulation were weighted as intact capsule and its content separately on electronic balance. The capsules are considered to be acceptable, if not more than 2 of the individual masses deviate from the average mass by more than the percentage deviation $10 \%$ and none deviate by more than $15 \%{ }^{19}$

\section{Microbiological evaluation of marketed CP brands and proniosomes filled capsules}

Two different brands of CP capsules $250 \mathrm{mg}$ were procured from the market and tagged as M1 and M2. Antimicrobial activity of optimized proniosomes and marketed CP capsules was performed by well diffusion method using agar nutrition media (Mueller Hinton Agar, MHA) against staphalococcus aureus microorganism. Inoculum having density of $10^{8} \mathrm{cfu} / \mathrm{mL}$ was prepared. McFarland 0.5 standard was obtained by mixing $0.05 \mathrm{~mL}$ of $1.175 \%$ barium chloride dihydrate $\left(\mathrm{BaCl}_{2} \cdot 2 \mathrm{H}_{2} \mathrm{O}\right)$, with $9.95 \mathrm{~mL}$ of $1 \%$ sulfuric acid $\left(\mathrm{H}_{2} \mathrm{SO}_{4}\right) \cdot{ }^{20}$ About $40 \mathrm{~mL}$ sterile agar along with the $100 \mu \mathrm{L}$ cultural suspension were poured evenly to each sterile prelabeled petri-plates. Plates were placed on flat surface for hardening. The cork borer was applied to bore a well of $10 \mathrm{~mm}$ diameter. Equal amount from the CP stock solution of test (F4S, F4M) and marketed (M1, M2) having $0.25 \%, \mathrm{w} / \mathrm{v}$ were transferred in each well respectively. 
The blank was also set without adding $\mathrm{CP}$ to one of the inoculated petri plate. The plates were then incubated at $37 \pm 2{ }^{\circ} \mathrm{C}$ for $24 \mathrm{hr}$ in biochemical oxygen demand incubator (B.O.D). Throughout the study, aseptic environment was maintained and the mean zone of inhibition was recorded and compared.

\section{RESULTS}

Formulation trials of $\mathrm{CP}$ proniosomes with different composition of surfactant and cholesterol blends were effectively obtained using sorbitol and maltodextrin carriers. Proniosomes were prepared as an alternative colloidal carrier approach, for controlled drug delivery system, maintaining the drug release for extended time period. These proniosomes were aqueously moistened $\left(60-65^{\circ} \mathrm{C}\right)$ to obtain niosomal vesicles directly. Upon microscopic evaluation, the vesicular systems were found to be lamellar, comprising of amphiphilic molecules surrounded by an aqueous environment. The prepared proniosomes were observed to be identical in dimension and shape. The structures of proniosomes and niosomes are shown in Figure 1 and 2, respectively.

Flowability and percent drug entrapment of various trials containing sorbitol (F0S to F8S) and maltodextrin (F0M to F4M) carriers were evaluated and are given in Table 2. In vitro drug release of proniosomes powder (F0M-F8M and F0S-F8S) was conducted using USP dissolution apparatus-I at 50rpm. Proniosomes containing sorbitol (F0S to F8S) and maltodextrin (F0M to F8M) carriers showed linear drug release with respect to time

\begin{tabular}{|c|c|c|c|c|c|}
\hline 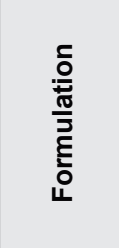 & 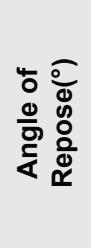 & 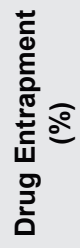 & 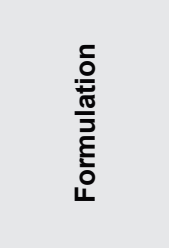 & 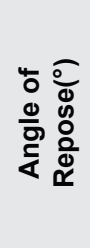 & 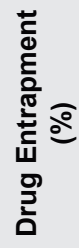 \\
\hline Sorbitol & 41.42 & - & Maltodextrin & 45.14 & - \\
\hline FOS & 36.70 & 48.45 & FOM & 39.80 & 52.12 \\
\hline F1S & 34.45 & 69.33 & F1M & 37.55 & 74.44 \\
\hline F2S & 32.54 & 74.05 & F2M & 36.23 & 79.92 \\
\hline F3S & 30.23 & 76.87 & F3M & 33. 47 & 84.22 \\
\hline F4S & 20.33 & 78.89 & F4M & 23.64 & 89.31 \\
\hline F5S & 27.45 & 69.44 & F5M & 30.41 & 77.35 \\
\hline F6S & 26.67 & 62.75 & F6M & 29.16 & 72.87 \\
\hline F7S & 24.38 & 58.12 & F7M & 27.41 & 65.19 \\
\hline F8S & 21.84 & 50.28 & F8M & 24.47 & 56.55 \\
\hline
\end{tabular}

(Figures 3 and 4). However, drug release was found to be delayed (up to $12 \mathrm{hr}$ ) in proniosomes containing higher levels of cholesterol (F7S, F8S and F7M, F8M) due to increase liphophilicity. F4M and F4S proniosomes were found to be optimized CP proniosomal formulations based on better \% drug entrapment efficiency, flowability and the drug release from vesicular system. Stability testing results showed only minor difference in drug remaining and drug entrapment efficiency during stability period between the formulations F4S and F4M (Table 3). Optimized proniosome (F4S and F4M) CP and marketed capsules (M1 and M2) were exposed to pharmaceutical quality control tests. The weight variation for F4S and F4M were found to be 280.9 (mg) \pm 1.15 and $317.3(\mathrm{mg}) \pm 1.27$, respectively, whereas for M1 and M2 were $270.9(\mathrm{mg}) \pm 5.32$ and $283.0(\mathrm{mg}) \pm 2.72$. Weight variation test complied with the pharmacopeial limits as no capsule deviate the filled weight by more than $10 \%$. Comparative microbiological evaluation of $\mathrm{CP}$ proniosomes with conventional capsules showed that optimized proniosomes were found to be more effective against Staphylococcus aureus rather conventional capsules. Furthermore, zone of inhibition was found to be small in initial hours with gradual increase up to $24 \mathrm{hr}$ (Table 4). This behavior of zone appearing primarily might be attributed to the controlled release of proniosomes capsules.

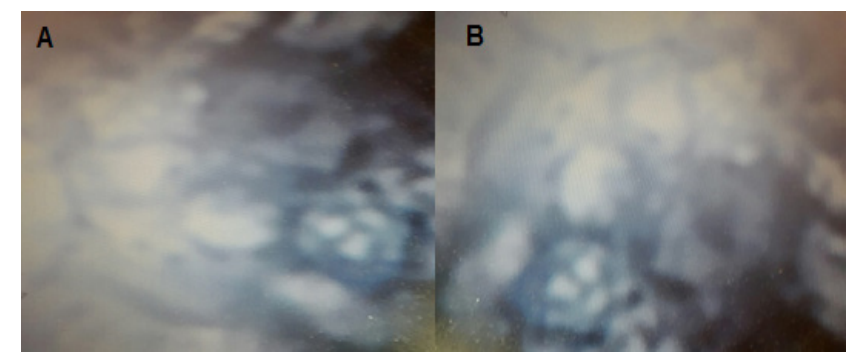

Figure 1: Optical microscopic view of powder proniosome (A) F4M (B) F4S

Table 3: Stability of optimized CP proniosome formulations (F4S and F4M) kept at controlled temperatures $\left(2-8^{\circ} \mathrm{C}\right)($ Mean $\pm \mathrm{SD}, n=3)$

\begin{tabular}{|c|c|c|c|c|}
\hline $\begin{array}{c}\text { Study } \\
\text { Period }\end{array}$ & $\begin{array}{c}\text { CP } \\
\text { Entrapment } \\
\text { Efficiency } \\
\%\end{array}$ & $\begin{array}{c}\text { \% CP } \\
\text { Remaining }\end{array}$ & $\begin{array}{c}\text { CP } \\
\text { Entrapment } \\
\text { Efficiency } \\
\%\end{array}$ & $\begin{array}{c}\% \text { CP } \\
\text { Remaining }\end{array}$ \\
\hline (Days) & F4S & F4S & F4M & F4M \\
\hline 0 & $78.89 \pm 0.19$ & $100.00 \pm 0.00$ & $89.31 \pm 0.22$ & $100.00 \pm 0.00$ \\
\hline 15 & $78.79 \pm 0.13$ & $99.78 \pm 0.14$ & $89.19 \pm 0.09$ & $99.38 \pm 0.17$ \\
\hline 30 & $78.75 \pm 0.10$ & $99.42 \pm 0.09$ & $89.12 \pm 0.12$ & $98.09 \pm 0.10$ \\
\hline 45 & $78.72 \pm 0.14$ & $99.13 \pm 0.18$ & $89.03 \pm 0.14$ & $97.89 \pm 0.15$ \\
\hline 60 & $78.65 \pm 0.22$ & $98.65 \pm 0.20$ & $88.85 \pm 0.18$ & $97.65 \pm 0.20$ \\
\hline 90 & $78.60 \pm 0.15$ & $98.24 \pm 0.19$ & $88.41 \pm 0.19$ & $97.44 \pm 0.12$ \\
\hline
\end{tabular}




\section{DISCUSSION}

$\mathrm{CP}$, a cephalosporin is the most prescribing antibiotic in health care system due to effectiveness against infections commonly occurring in population. ${ }^{21}$ In the present study, CP proniosomes were successfully prepared using cholesterol, span 60 and carriers to provide controlled drug delivery. Proniosomes are the vesicular system with improved penetration designed to prolong the drug duration and reduction in dosing frequency. Hence forth, providing drug safety and better patient compliance. Maltodextrin and sorbitol are advantageous carriers in the preparation of proniosomes as documented to be

\begin{tabular}{|c|c|c|c|c|}
\hline opti & $\begin{array}{l}\text { ble } 4 \\
\text { ized }\end{array}$ & $\begin{array}{l}\text { mparative b } \\
\text { oroniosomal } \\
\text { (Mean } \pm S\end{array}$ & $\begin{array}{l}\text { ctericid } \\
\text { and mar } \\
, n=3 \text { ) }\end{array}$ & adivity of \\
\hline S. No & Sample & $\begin{array}{c}\text { Drug } \\
\text { concentration } \\
(w / v)\end{array}$ & $\begin{array}{c}\text { Quantity } \\
\text { of } \\
\text { sample }\end{array}$ & $\begin{array}{c}\text { Zone of } \\
\text { inhibition at } \\
24 \mathrm{~h} \\
(\mathrm{~mm})\end{array}$ \\
\hline 1 & F4S & \multirow{4}{*}{$0.25 \%$} & \multirow{4}{*}{$0.1 \mathrm{~mL}$} & $31.20 \pm 0.19$ \\
\hline 2 & F4M & & & $34.4 \pm 0.23$ \\
\hline 3 & M1 & & & $17.2 \pm 0.34$ \\
\hline 4 & M2 & & & $19.4 \pm 0.27$ \\
\hline
\end{tabular}

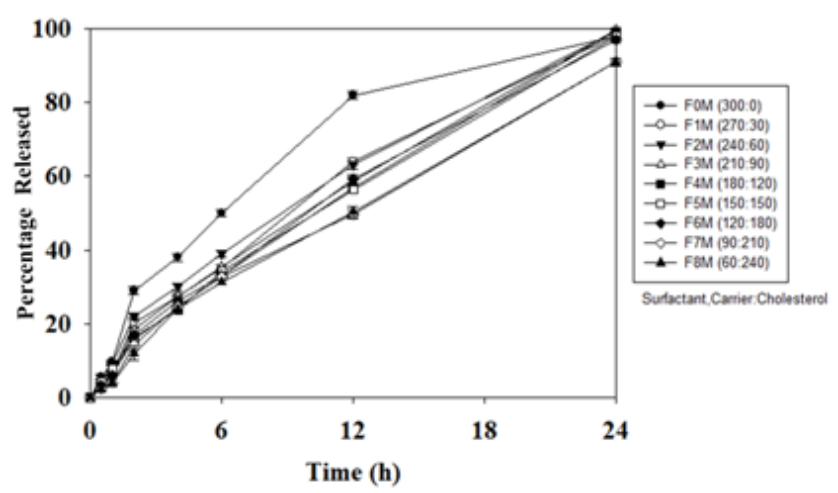

Figure 2: $\mathrm{CP}$ release from proniosomes with maltodextrin carrier (FOM to F8M)

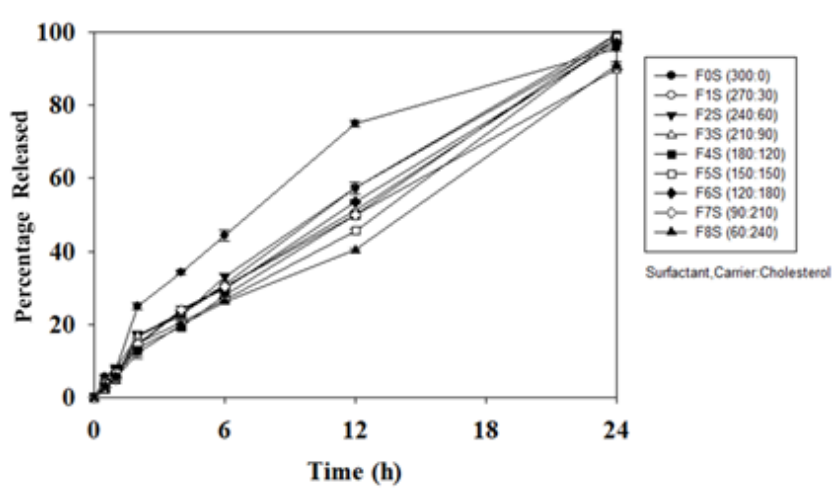

Figure 3: CP release from proniosomes with sorbitol carrier (F0S to F8S) biocompatible, biodegradable, non-carcinogenic and non-immunogenic. Moreover, nonionic surfactant and cholesterol are beneficial as improving the efficacy and stability of the encapsulated active moities. ${ }^{22,23}$ Currently, span 60 and cholesterol are used to facilitate stable vesicle formation, enhance the encapsulation of the drug thus improving the oral delivery of CP from proniosomes. Not only the selection of surfactant and lipids, rather are their quantities considered to be critical during formulation development of pronisomal system. Alteration in the lipid composition could cause deformation of the vesicle leading to drug leakage prior to diffusion and fusion of vesicle with gastrointestinal epithelium, resulting in less drug entrapment or abrupt drug release. ${ }^{24-28}$ Based on these facts, various ratios of span 60 with cholesterol were tried and the responses against variables were studied to achieve optimized formulation(s).

Not only the formulation ingredients but the employed technique for the preparation plays a significant role in the successful development of a product. Currently, CP proniosomes were developed using slurry method owing to benefits as discussed in past studies. ${ }^{13,15}$ The method provides uniform, consistent and thinner coating with higher surface area facilitating the rehydration with better stability of vesicles. Better entrapment of CP was observed in all formulations containing span 60 probably due to longer chain length resulting in larger

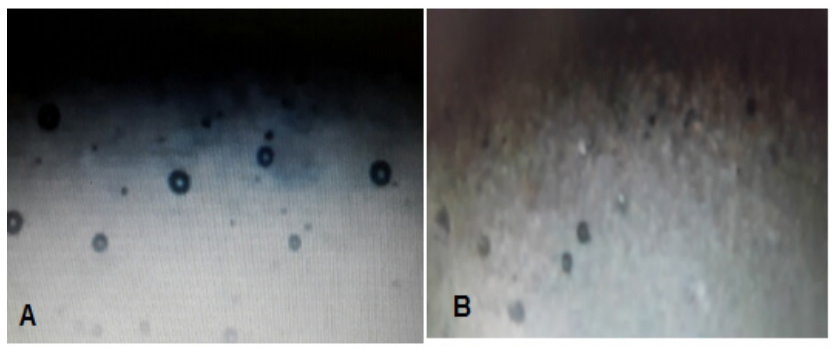

Figure 4: Optical microscopic view of niosomal dispersion (A) F4M (B) F4S

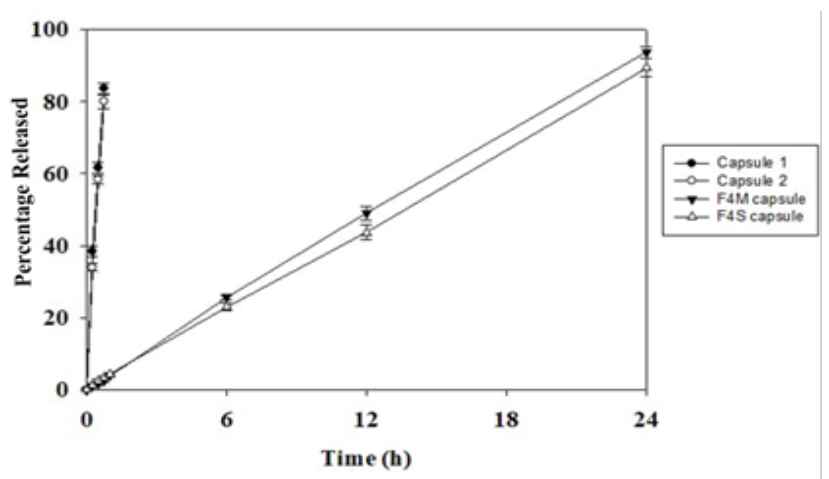

Figure 5: Drug release from the CP capsules marketed brands and proniosomal (optimized formulations of sorbitol and maltodextrin) 
core space. Additionally, cholesterol influenced the physical properties and vesicle structures by modulating cohesion and mechanical strength of bi-layers and consequently leading to higher entrapment of the drug. The CP entrapment efficiency was found to be higher in F3S to F5S and F3M to F5M proniosomes. Similar findings were reported by Nagalakshmi and coworkers, concluding that the entrapment of the drug was greater in formulation containing approximately equal ratios of surfactant to cholesterol than other experimental runs. ${ }^{29}$ Low entrapment of CP was noticed in FOS and FOM trials due to the absence of cholesterol therefore, speedy drug release $>75 \%$ was obtained in $12 \mathrm{hr}$. While, moderately higher concentration of cholesterol was observed to entrap CP efficiently (F1S to F4S and F1M to F4M). However, decline in drug entrapment was noticed at higher concentration of cholesterol (F5S to F8S and F5M to $\mathrm{F} 8 \mathrm{M}$ ) leading to decrease $\mathrm{CP}$ entrapment in the vesicles (69.44 to $50.28 \%$ and 77.35 to $56.55 \%$, respectively). This reflects the fact that cholesterol is prone to make changes in the normal bi-layered structure, resulting in reverse drug entrapment. Excess of cholesterol was unsuitable, as equal molarities of nonionic surfactant and cholesterol might build the membrane dense and ordered. ${ }^{30}$ Mokhtar Ibrahim et al. found that increase in cholesterol after certain level would in turn lower the drug entrapment efficiency. ${ }^{31}$ Actually, cholesterol (above the saturation edge) took the places in the bilayer membrane with its $\mathrm{OH}$ moiety (bilayer packing) thus preventing the $\mathrm{CP}$ to be entered in the bilayer leading to turn down the $\mathrm{CP}$ entrapment. ${ }^{32,33}$

Proniosomes prepared using sorbitol (F0S to F8S) and maltodextrin (FOM to F8M) carriers showed a comparable drug release profile as presented in Figure 2 and 3, respectively. This indicates that both carriers can be successfully used to prepare CP proniosomes. Drug release was found to be mainly governed by the blend ratio of surfactant and membrane stabilizer.

In the present study, niosomes prepared by aqueous hydration of proniosomes were found to be discrete vesicles (without aggregation or lumping) with uniform spherical shape and smooth surface. It is also documented in literature that the niosomes prepared from proniosomes are superior than conventional niosomes in term of their physical and chemical stability. ${ }^{23,34}$ Song et al. ${ }^{35}$ had also encapsulated Vinpocetine-a synthethic alkaloid in proniosomes resulting in higher gastrointestinal absorption ultimately leading to increase in drug bioavailability with improved stability.

Proniosomes being a successful drug delivery system opens a new horizon for the pharmaceutical industries. Such systems are offering promising benefits due to better flowability and stability of encapsulated drugs. Proniosomes, being dry powders are easily fabricated into oral, transdermal and parenteral preparations. ${ }^{36,37}$ Tablets and capsules are among the popular oral dosage forms as providing advantages over other drug administration routes due to self and convenient administration. In this connection, presently the optimized formulations of $\mathrm{CP}$ proniosomes (F4S and $\mathrm{F} 4 \mathrm{M}$ ) Figure 5 were filled in hard gelatin capsules. These CP proniosome filled capsules were then compared with two conventional marketed brands (M1 and M2) of CP capsules having same strength $(250 \mathrm{mg})$. Antimicrobial efficacy of the proniosomes was compared with the reference products through well method. Greater zone of inhibition was observed around F4S and F4M proniosomes against Staphylococcus aureus reflecting their better activity than the marketed CP capsules. Similar findings were reported by Kumar and coworkers presenting higher zone of inhibition with cephalosporin niosomes in contrast to the marketed formulation. ${ }^{38}$ Thus, the CP proniosomes have been successfully obtained by this study is considered to be superior than conventional dosages owing to longer drug stay with controlled drug release and, improved stability and efficacy.

\section{CONCLUSION}

Cephardine proniosomes were dry free flowing powders prepared successfully using surfactant, cholesterol and carriers (sorbitol/ maltodextrin). F4M and F4S containing blend of span 60 and cholesterol in ratio of 180:120 were found to be optimized proniosomal trials. Optimized formulations were stable showing negligible change in drug content and entrapment efficiency over study period. Being lipid based vesicular system, the designed proniosomes prolong the duration of the drug in systemic circulation thus reducing the toxicity by selective up taking. The optimized proniosomes enhances the antimicrobial activity of the drug with its sustained effect consequently, improving the patient compliance.

\section{ACKNOWLEDGEMENT}

The authors would like to acknowledge the kind support of from the Board and Advanced Studies and Research (BASR), Federal Urdu University, Karachi.

\section{CONFLICT OF INTEREST}

The authors declare no conflict of interest. 


\section{ABBREVIATIONS}

CP: Cephradine.

\section{REFERENCES}

1. Kumar SP, Kumar NS, Raghuveer I. Proniosome as a sustained drug delivery system: a review. Int Res J Pharm. 2012;3:27-32.

2. Bandopadhyay S, Manchanda S, Chandra A, Ali J, Deb PK. Overview of different carrier systems for advanced drug delivery. In: Tekade RK, editor. Drug delivery systems. Cambridge: Academic Press; 2020. p. 179-233.

3. Patel R, Prabhu P. Nanocarriers as versatile delivery systems for effective management of acne. Int J Pharm. 2020;579:119140. doi: 10.1016/j. ijpharm.2020.119140.

4. AIQahtani AD, O'Connor D, Domling A, Goda SK. Strategies for the production of long-acting therapeutics and efficient drug delivery for cancer treatment. Biomed Pharmacother. 2019;113:108750. doi: 10.1016/j. biopha.2019.108750.

5. Langer RS, Wise DL. In: Langer RS, Wise DL, editors. Medical applications of controlled release. OH: Taylor \& Francis, CRC Press; 2019.

6. Supraja B, Mulangi S. An updated review on pharmacosomes, a vesicular drug delivery system. J Drug Delivery Ther. 2019;9(1-s):393-402. doi: 10.22270/jddt.v9i1-s.2234.

7. Dhadwal A, Sharma DR, Pandit V, Ashawat MS, Kumar P. Cubosomes: Anovel carrier for transdermal drug delivery. J Drug Delivery Ther. 2020;10(1):123-30. doi: 10.22270/jddt.v10i1.3814.

8. Bhardwaj P, Tripathi P, Gupta R, Pandey S. Niosomes: a review on niosomal research in the last decade. J Drug Deliv Sci Technol. 2020;56. doi: 10.1016/j. jddst.2020.101581, PMID 101581.

9. Ruckmani K, Sankar V, Sivakumar M. Tissue distribution, pharmacokinetics and stability studies of zidovudine delivered by niosomes and proniosomes. J Biomed Nanotechnol. 2010;6(1):43-51. doi: 10.1166/jbn.2010.1101, PMID 20499831.

10. Yasam VR, Jakki SL, Natarajan J, Kuppusamy G. A review on novel vesicular drug delivery: proniosomes. Drug Deliv. 2014;21(4):243-9. doi: 10.3109/10717544.2013.841783, PMID 24128089.

11. Farooqui N, Jaiswal V, Kar M. A review on proniosomal gel: potential carrier system in transdermal delivery for non-steroidal anti-inflammatory drugs (NSAID). Int J Pharm Life Sci. 2014;5:3939-47.

12. Alsarra IA, Bosela AA, Ahmed SM, Mahrous GM. Proniosomes as a drug carrier for transdermal delivery of ketorolac. Eur J Pharm Biopharm. 2005;59(3):485-90. doi: 10.1016/j.ejpb.2004.09.006, PMID 15760729.

13. Naggar VF, El Gamal SS, Allam AN. Proniosomes as a stable carrier for oral acyclovir: formulation and physicochemical characterization. J Am Sci. 2012;8:417-28.

14. Pawar PS, Saleem M. Formulation and evaluation of oral colon targeted tablet of budesonide. Pharm Lett. 2013;5:1-12.

15. Sammour RMF, Taher M, Chatterjee B, Shahiwala A, Mahmood S Optimization of aceclofenac proniosomes by using different carriers, Part 1: Development and characterization. Pharmaceutics. 2019 Jul;11(7):350. doi: 10.3390/pharmaceutics11070350, PMID 31323799.

16. Naveed S, Jaweed L. UV spectrophotometric assay of different brands of cephradine. Health Sci Res. 2014;1:84-7.

17. Chauhan MK, Sahoo PK, Rawat AS, Duggal D, Kandwal M, Sandal N Formulation, characterization and in vitro evaluation of tactically engineered proniosomes for successful oral delivery of ramipril. Pharm Lett. 2015;7:93-7.

18. Ertekin ZC, Bayindir ZS, Yuksel N. Stability studies on piroxicam encapsulated niosomes. Curr Drug Deliv. 2015 Apr 1;12(2):192-9. doi: 10.2174/156720181 1666140723115852, PMID 25056419.

19. British Pharmacopoiea. London, UK: Her Majesty's Stationery Office, BMJ Group and Phrarmaceutical Press; 2018.
20. McFarland J. The nephelometer: an instrument for estimating the number of bacteria in suspensions used for calculating the opsonic index and for vaccines. J Am Med Assoc. 1907;49:1176-8.

21. Machado-Alba JE, Valladales-Restrepo LF, Gaviria-Mendoza A, MachadoDuque ME, Figueras A. Patterns of antibiotic prescription in Colombia: are there differences between capital cities and municipalities? Antibiotics (Basel). 2020;9(7):389. doi: 10.3390/antibiotics9070389, PMID 32650491.

22. Sadeghi-Ghadi Z, Ebrahimnejad P, Talebpour Amiri F, Nokhodchi A. Improved oral delivery of quercetin with hyaluronic acid containing niosomes as a promising formulation. J Drug Target. 2021;29(2):225-34. doi: 10.1080/1061186X.2020.1830408. PMID 32997536.

23. Raeiszadeh M, Pardakhty A, Sharififar F, Farsinejad A, Mehrabani M, HosseiniNave H, Mehrabani M. Development, physicochemical characterization, and antimicrobial evaluation of niosomal myrtle essential oil. Res Pharm Sci. 2018;13(3):250-61. doi: 10.4103/1735-5362.228955, PMID 29853934.

24. Hu C, Rhodes DG. Proniosomes: a novel drug carrier preparation. Int J Pharm. 2000;206(1-2):110-22. doi: 10.1016/S0378-5173(99)00122-2, PMID 11202988.

25. Solanki AB, Parikh JR, Parikh RH. Formulation and optimization of piroxicam proniosomes by 3-factor, 3-level Box-Behnken design. AAPS PharmSciTech. 2007;8(4):E86. doi: 10.1208/pt0804086, PMID 18181547.

26. Ogiso T, Niinaka N, Iwaki M. Mechanism for enhancement effect of lipid disperse system on percutaneous absorption. J Pharm Sci. 1996;85(1):57-64. doi: 10.1021/js950178x, PMID 8926585.

27. Kumar S, Jain PK, Pandey N, Saxena G. Comparative study of proniosomal drug delivery system of flurbiprofen. J Chem Pharm Res. 2016;8:222-8.

28. Kumar S, Gupta R, Prajapati S, Gupta N, Pattnaik S, Agrawal A. Proniosomes formulation and evaluation by slurry method as an emerging provesicular drug carrier in NDDS. World J Pharm Pharm Sci. 2014;3:1486-501.

29. Nagalakshmi S, Krishnaraj K, Jothy AM, Chaudhari PS, Pushpalatha H, Shanmuganthan S. Fabrication and characterization of herbal drug-loaded nonionic surfactant based niosomal topical gel. J Pharm Sci Res. 2016;8:1271.

30. Hao Y, Zhao F, Li N, Yang Y, Li K. Studies on a high encapsulation of colchicine by a niosome system. Int J Pharm. 2002;244(1-2):73-80. doi: 10.1016/s03785173(02)00301-0, PMID 12204566.

31. Mokhtar Ibrahim M, Tawfique SA, Mahdy MM. Liposomal diltiazem $\mathrm{HCl}$ as ocular drug delivery system for glaucoma. Drug Dev Ind Pharm. 2014;40(6):765-73. doi: 10.3109/03639045.2013.783589, PMID 23570512.

32. Toshimitsu Y, Florence AT. Vesicle (niosome)-in-water-in-oil (v/w/o) emulsions: an in vitro study. International Journal of Pharmaceutics. 1994;108(2):117-23. doi: 10.1016/0378-5173(94)90322-0.

33. Rahman MA, Yusuf M, Alshammari T, Faiyazuddin M. Nanopharmaceuticals. In: Relevance to drug delivery and targeting. Nanopharmaceuticals: principles and applications. NY: Springer; 2020. p. 77-112.

34. Boddu M, Choppari V, Rapalli V, Badam M. Formulation and evaluation of proniosomes of felodipine. Drug Des. Vol. 6; 2017. PMID 1000154.

35. Song S, Tian B, Chen F, Zhang W, Pan Y, Zhang Q, Yang X, Pan W. Potentials of proniosomes for improving the oral bioavailability of poorly water-soluble drugs. Drug Dev Ind Pharm. 2015;41(1):51-62. doi: 10.3109/03639045.2013.845841, PMID 24111828

36. Emad Eldeeb A, Salah S, Ghorab M. Proniosomal gel-derived niosomes: an approach to sustain and improve the ocular delivery of brimonidine tartrate; formulation, in-vitro characterization, and in-vivo pharmacodynamic study. Drug Deliv. 2019;26(1):509-21. doi: 10.1080/10717544.2019.1609622, PMID 31090464.

37. Kapoor B, Gupta R, Gulati M, Singh SK, Khursheed R, Gupta M. The Why, Where, Who, How, and What of the vesicular delivery systems. Adv Colloid Interface Sci. 2019;271:101985. doi: 10.1016/j.cis.2019.07.006.

38. Nair SC, Kumar BS, Krishna R, Ps L, Vasudev DT. Formulation and evaluation of niosomal suspension of cefixime. Asian J Pharm Clin Res. 2017;10(5):194-201. doi: 10.22159/ajpcr.2017.v10i5.17189. 


\section{SUMMARY}

Proniosomes powders loaded with cephradine (CP) have been prepared using span60 and cholesterol with maltodextrin (F0M-F8M) and sorbitol (F0S-F8S) as a carrier. Various formulations prepared were characterized for powder flowability, drug entrapment efficiency, and in vitro drug release. It has been found that formulations F4S and F4M possess good flowability, excellent drug entrapment, and sustained drug release. These two formulations were also compared with the marketed conventional capsule brands for microbiological efficacy. It has also been found that the prepared proniosomes are highly stable with the drug efficiency (\%) of $78.60 \pm 0.15$ and $88.41 \pm 0.19$ for F4M and F4S, respectively, and they possess higher bactericidal activities against $S$. aureus than the reference (M1 and M2).

\section{About Authors}

Dr. Zubair Anwar is currently working as Assistant Professor in the Department of Pharmaceutical Chemistry in Faculty of Pharmaceutical Sciences of Baqai Medical University, Karachi. He obtained Ph. D. degree in Pharmaceutical Chemistry from Baqai Medical University and conducted Postdoctoral research at the Adam Mickiewicz University, Poznan on a fellowship awarded by National Science Centre of Poland.

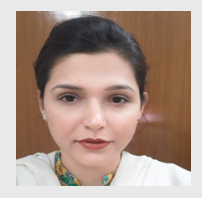

Dr Sana Ghayas is currently working as Assistant Professor in the Department of Pharmaceutics in Faculty of Pharmaceutical Sciences of Dow Medical University, Karachi. She obtained her Ph. D. degree in Pharmaceutics from the Faculty of Pharmacy of the University of Karachi, Karachi.

Cite this article: Saim MAF, Bashir L, Naz S, Ghayas S, Bushra R, Anwar Z, Uddin SA, Korai U. Development and Characterization of Cephradine Proniosomes for Oral Controlled Drug Delivery. Indian J of Pharmaceutical Education and Research. 2022;56(1s):s67-s74. 